\title{
Vinyl chloride diseases
}

\author{
MALCOLM I. V. JAYSON \\ From the Department of Medicine, University of Bristol, and Royal National Hospital for \\ Rheumatic Diseases, Bath
}

With ever increasing consumption and diminishing reserves of raw materials such as iron, copper, lead, and other minerals increasing demands are being made for plastics, and of these polyvinyl chloridePVC - has proved one of the most important. PVC is made (Barnes, 1976) by polymerizing the monomer vinyl chloride (VC), which is normally gaseous under atmospheric pressure. VC is pumped into a reactor under pressure as a suspension in water together with a catalyst and suspending agent. When heated to the appropriate temperature the vinyl chloride molecules condense to form long chain molecules of PVC which precipitate. The fluid from the reactor is drained as a slurry which is centrifuged to separate the PVC from waste water. After further cleaning and drying the PVC is bagged and ready for use. In contrast with continuous flow processes this is a batch method of production.

The reactor is filled with materials and polymerization takes place. The reactor is then emptied and cleaned and the cycle is restarted. This is because PVC is deposited on the side walls of the chamber and is a poor conductor of heat. Since polymerization generates much heat the temperature would rise too high if the PVC were not removed. Cleaning of the side walls between each batch therefore is essential, and before the dangers of vinyl chloride became known this was performed manually by workers, known as 'poly-cleaners', who climbed inside the reactor. Although the chamber was ventilated and the men should have worn masks there is little doubt that they were exposed to high concentrations of VC monomer.

\section{Acro-osteolysis}

Suciu et al. (1963) first described Raynaud's phenomenon and scleroderma in VC workers. They found these in about $6 \%$ and $3.6 \%$ of subjects respectively. Subsequently, Cordier et al. (1966), Harris and Adams (1967), and others have described the particular association of acro-osteolysis with VC exposure. Dinman et al. (1971) found 25 definite and 16 possible cases of acro-osteolysis among 5011 employees in PVC factories, and Wilson et al. (1967) and Cook et al. (1971) showed that the condition is specifically associated with $\vec{\omega}$ poly-cleaning. Levels in the reactor were about 50 ppm but close to the surfaces from where the PVC was being scraped they were up to $1000 \mathrm{ppm}$.

Raynaud's phenomenon precedes the bone and skin changes (Dodson et al., 1971) and objective measurements show interference with the peripheral circulation (Dodson et al., 1971; Lilis et al., 1975; Veltman et al., 1975).

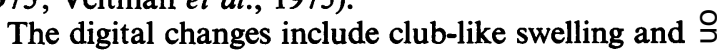
shortening of the terminal phalanges with shorten- $\rightarrow$ ing of the nails. The skin becomes thickened and coarsened, often with localized placques and areas of induration. Biopsy shows excessive amounts of dermal collagen which may resemble the changes of scleroderma.

Osteolysis presents a characteristic radiologica appearance in the terminal phalanges of the fingers (Fig. 1) with transverse dissolution of the central parts of the bone but often with faint residual transverse opacities (Veltman et al., 1975; Preston

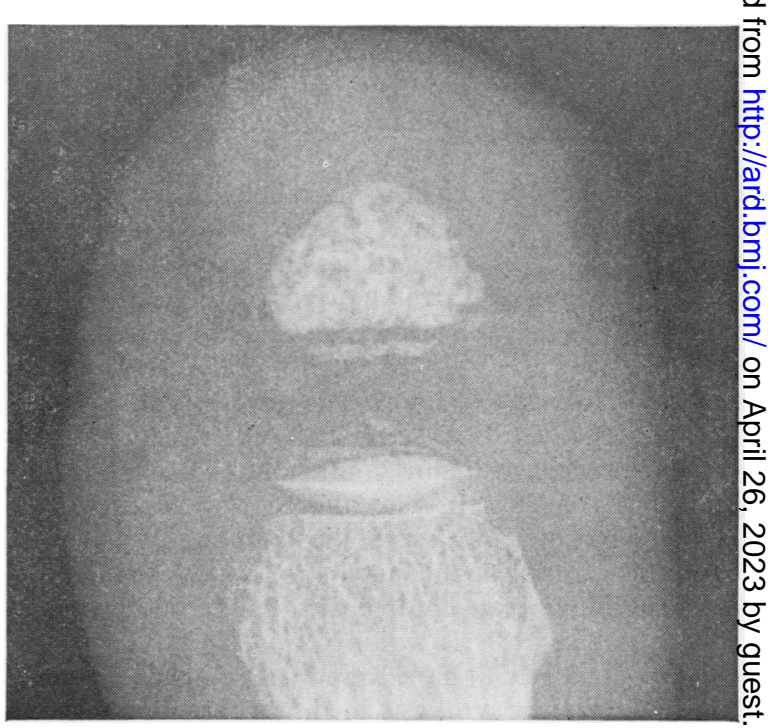

FIG. 1 Terminal phalanx showing transverse dissolution in central part with some residual transverse opacities 


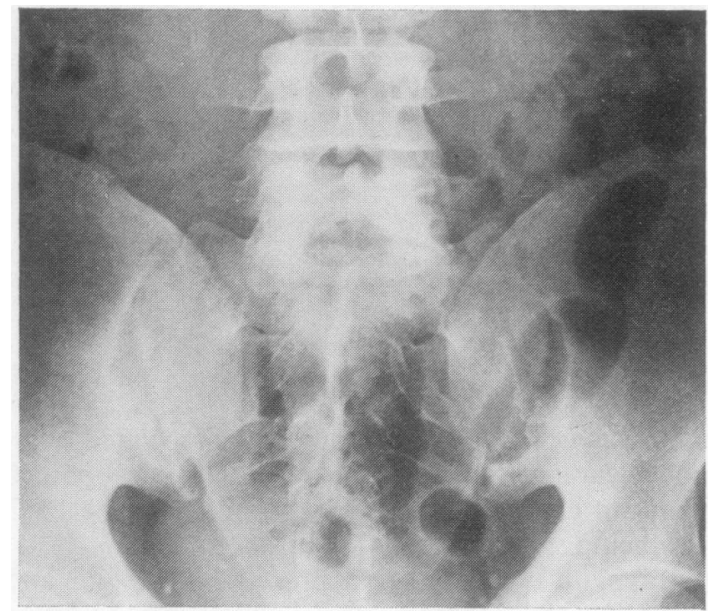

FIG. 2 Sacroiliac joints showing lysis of iliac sides of joints

et al., 1976). Lytic areas may also occur in bones elsewhere, including the long bones, the mandible, and the phalanges of the toes. Areas of lysis are also found on the iliac side of the sacroiliac joints but the changes do not resemble ankylosing spondylitis (Fig. 2).

Oesophageal involvement, which is common in systemic sclerosis, does not occur in this condition (Dodson et al., 1971), but chest $x$-ray examination may show fibrotic changes (Lilis et al., 1975). Indeed, changes in pulmonary function have been observed in VC workers without skin changes (Lange et al., 1974; Miller et al., 1975).

Lange et al. (1974) found no abnormalities in antinuclear factor, rheumatoid factor, or LE cells, but Ward et al. (1976) found evidence of soluble immune complexes in 20 out of 58 PVC workers. Their incidence closely paralleled the clinical features of the disease. There was evidence of complement activation via the classical pathway and immunofluorescent examination of affected tissues showed deposition of $1 \mathrm{gG}, \mathrm{C}_{3}, \mathrm{C}_{4}$, fibrin, and fibrinogen in the walls of small blood vessels. Ward et al. (1976) suggest that VC is metabolized to form a highly reactive dioxide which is either incorporated into proteins producing conformational changes or can act as a hapten. The altered protein provokes the formation of antibodies and subsequently immune complexes. These are deposited in the vessel wall, activate complement, and could provoke collagen biosynthesis leading to vascular occlusion. Jayson et al. (1976) have found preliminary evidence of new collagen synthesis in the skin mimicking the changes in scleroderma. They demonstrated excess reducible cross-links and measured the rate of incorporation of tritiated proline into hydroxy- proline in skin in organ culture. Viola (1970) produced pathological changes in collagen and connective tissue resembling acro-osteolysis by exposing rats to high concentrations $(30000 \mathrm{ppm})$ of VC.

\section{Liver and spleen lesions}

Viola (1971) reported the development of tumours in animals exposed to high concentrations (30000 ppm) of vinyl chloride. Maltoni and Lefemine (1975) found that malignancy could be produced at much lower levels $(50 \mathrm{ppm})$. Such studies provoked a search for liver tumours in human VC workers, and almost immediately Creech and Johnson (1974) found three cases of angiosarcoma of the liver. Since then there have been further reports of such tumours (Lee and Harry, 1974; Falk et al., 1974; Popper and Thomas, 1975). Moreover, Monson et al. (1974) found a 50 per cent increase in deaths from cancer as a whole in VC workers.

Surveys of PVC workers show a high incidence of hepatosplenomegaly. Some have abnormal liver function tests (Marsteller et al., 1973; Duck, 1976) and degenerative changes in hepatocytes with collagenization of the sinusoidal walls and septal fibrosis are found on biopsy. There may be activation and proliferation of atypical sinusoidal cells (Gedigk et al., 1975; Marsteller et al., 1975), which may precede the development of the angiosarcoma (Heath et al., 1975; Popper and Thomas, 1975). The pathology of the tumours has been described by Thomas and Popper (1975) and Weinbren (1976). The lesions are very similar to those that follow chronic exposure to arsenicals and thorotrast.

\section{Other problems}

Funes-Cravioto et al. (1975) and Purchase et al. (1976) found an increased number of chromosomal abnormalities in people exposed to VC. This may indicate genetic hazards, and an excessive number of fetal deaths were found to occur in the pregnancies of women whose husbands had been exposed to the chemical (Infante et al., 1976). It seems from animal experimentation that vinyl chloride is mutagenic only after metabolic activation in the liver (Rannug et al., 1974). This may be relevant to its carcinogenicity and explain why tumours particularly occur in the liver.

Thrombocytopaenia has also been found in many patients exposed to vinyl chloride (Veltman et al., 1975). This may be related to the peripheral vascular disease. A wide variety of other symptoms have been reported in PVC workers. They range from excessive fatigue, somnolence, and aches in bones and muscles to loss of libido (Walker, 1976), but it is difficult to evaluate their true significance. 


\section{Conclusions}

All these syndromes are particularly associated with exposure to high concentrations of VC. Other workers in the same factories have shown little if any evidence of these diseases. Reactor cleaning is now done externally, avoiding the need for workers to enter the chamber. There is much improved control over leaks into the environment together with considerable lowering of the acceptable limits of concentrations in the atmosphere. The methods for environmental monitoring have been dramatically improved so that small leaks are immediately $\stackrel{\vec{\rho}}{\rightarrow}$ detected. Close medical surveillance of all VC workers is now routinely performed. We must hope that these measures will result in the eradication of $\frac{\bar{c}}{\sigma}$ diseases due to vinyl chloride. 